\title{
A regional model for urban sustainability: emerging innovation clusters within knowledge societies - MENA region case
}

\author{
M. Shokry ${ }^{1,2}$ \\ ${ }^{1}$ Architecture Department, College of Architecture and Design, \\ Effat University, Saudi Arabia \\ ${ }^{2}$ Architecture Department, College of Engineering, \\ Cairo University, Egypt
}

\begin{abstract}
The global context of urbanization, urges the dramatic spread of gradual accumulation of human resources, economic powers and political responsibilities within cities, the contemporary context presents an urgent impetus to evaluate how cities can lead and innovate. The world is entering a new cycle of development, with the economic crisis and restructuring of 2007-2010 largely over and new energy infusing the investment and sustainable development agenda once more. The first part of the paper is trying to establish some criterion of linking sustainable development mode of the developing world societies with type of knowledge transfer and exchange within regions through a group of clusters or innovation hubs. Also it's trying to prove that the typology of knowledge production centers (science and technology parks, innovation hubs, research parks, etc.) can be a sustainable flexible model to be adopted in different types of communities. The second and third parts are paying more attention on the aspects of sustainability within knowledge and innovation clusters: definitions, how they can emerge, flourish or collapse within a dynamic flow of knowledge, people and ideas worldwide. The final part sheds some lights on the case of the MENA region and its various societies. Starting by classifying these regions acceding to its knowledge capacity index (KCI). It's also keen to come to grips with the major problems that afflict sustaining knowledge society in MENA societies, whether in research, education, or the use of the new technological mediums that underlie today's knowledge networks.

Keywords: innovation clusters, knowledge exchange, urban policy, MENA, science parks, knowledge societies.
\end{abstract}




\section{Introduction}

According to Almeida et al. [1], the science and technology parks (STP), technopoles, innovation centres or knowledge hubs are considered as the platforms to establish a set of links between market firms (business) and universities (academia) to provide access to knowledge through a range of administrative, logistic and technical services and to foster technology transfer to the outer community in different regional levels to maintain a balanced, sustainable and integrated development nationwide.

In the last century, a certain typology of STP stared with some typical spatial configurations, such as a cluster-type planned property, including a group of buildings near from university campus, low ratio of building's footprint and high quality design within a park-like landscaping.

More recently, the STP developed to be a hotspot within the city acts as a wellplanned innovative milieu comprising firms with high level of competitiveness. This dramatic change in the conceptual and spatial configuration of STP and other innovation hubs is only the first sign for more and more transformations of this type of projects to stand with the dramatic shifts of our booming knowledge society.

Moreover, the paradigms shift from the "linear" concept of innovation, is turning to act as an "integral" approach to involve the whole community in the process of knowledge production and exchange within a sustainable process and environment.

While Enright and Roberts [2] argue that trends towards globalization of industries and companies might appear to reduce the importance and distinctiveness of (sub-national) regions, a tendency towards localization of certain industries and economic activities appears to do exactly the opposite. The simultaneous globalization and localization tendencies have created policy challenges for national and local governments.

\section{Learned lessons: knowledge exchange models}

Throughout the last decade, patents, applied research, knowledge production, recycling and exchange, in addition to technology transfer are considered the key engines of innovation in industrialized countries, especially where education systems are well developed, and universities produce a wealth of innovative solutions through basic and applied research. The concepts of 'technology' and 'transfer' can be described with the words of Gibson and Rogers [3]:

"The concepts of technology and of transfer are defined by both theoreticians and practitioners in many different ways. There is usually agreement, however, that (1) technology is not just a 'thing', and (2) transfer is a profoundly human endeavor. Essentially, 'technology' is information that is put to use in order to accomplish some task, the knowledge of how to do something. 'Transfer' is the movement of technology via some channel from one individual or organization to another. " Currently, three models of innovation systems are applied globally: 


\subsection{The classical knowledge exchange model}

US and EU regions applied the "stage model" which has been developed by Gibson et al. [3] differentiates the process of knowledge transfer in different stages by different criteria for success, taking into account the role and function of the actors. As a stage model it allows also insights into the procedural nature of knowledge transfer. It therefore represents an important basis for understanding the process of transfer of knowledge both with regard to the interaction of the actors as well as to its temporary dimension.

This model represents no linear process of knowledge transfer, which takes place gradually from level one to four. Each step in itself represents a kind of knowledge transfer, which differs from one another by its success criteria. The complexity of transfer increases with each step. A collaboration of knowledgegiver and knowledge-recipient is needed mainly in the second and third stages 'acceptance' and 'implementation' of research results, while in stage one and four the 'generation' and 'application' of research results each actor is responsible on his own.

De Prato and Nepelski [5] argues that this model is considered the most successful one with emerging fears of lagging in US, while In Europe, knowledge and technology transfer between university and industry is still a missing link in the innovation value chain. Among the factors that have hindered the development of university-industry partnerships and academic spinoffs, the most evident are the absence of an entrepreneurial culture in many European universities, the lack of a full-fledged, pan-European patent and the limited development of innovation markets and intermediaries.

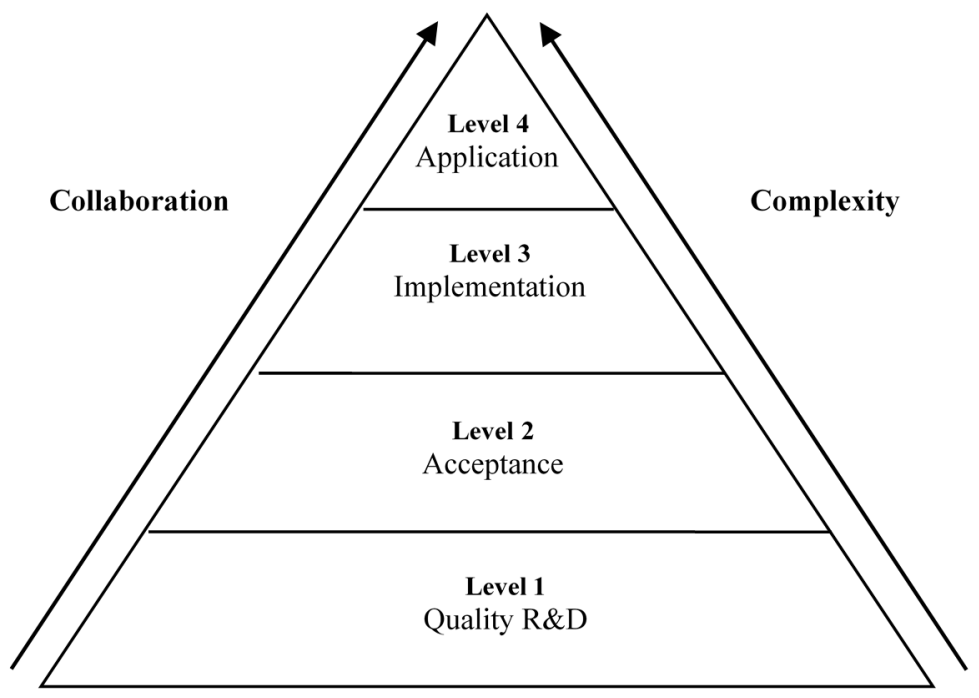

Figure 1: "Stage model” of knowledge exchange. Source: Gibson and Rogers (1994:335). 


\subsection{A dynamic knowledge exchange model}

According to Bozeman [6], knowledge or technology transfer between the public and private sector is a complex process that depends on the involved actors:

1. The transfer agent, the research institution that disseminates the research results,

2. The transfer recipient, the company that tries to incorporate the research results in their own products,

3. The constitution of the transfer object, e.g. the knowledge to be transferred,

4. The transfer channel through which the results are disseminated, and

5. The economic environment (innovation ecosystem) in which the transfer takes place.

This model is therefore suitable as a starting point for developing an own model of knowledge transfer to analyse the effectiveness of transfer barriers.

\subsection{An interactive-recursive model of knowledge transfer}

The real shortcomings the stage model and the dynamic knowledge exchange model are the lack of interaction specification of the knowledge transfer actors involved. And the interactivity of the process involved in knowledge transfer.

The interactive-recursive model of knowledge transfer is basically depending on interest in knowledge. It can be considered as a comprehensive model by the establishment of three basic dimensions and the connection of each dimension with a group of actors that represent the variables of the model. The particular group of actors is defined by their participation in the respective dimension. The composition of the major players involved in each dimension can vary depending on the type of knowledge transfer from case to case (Figure 2).

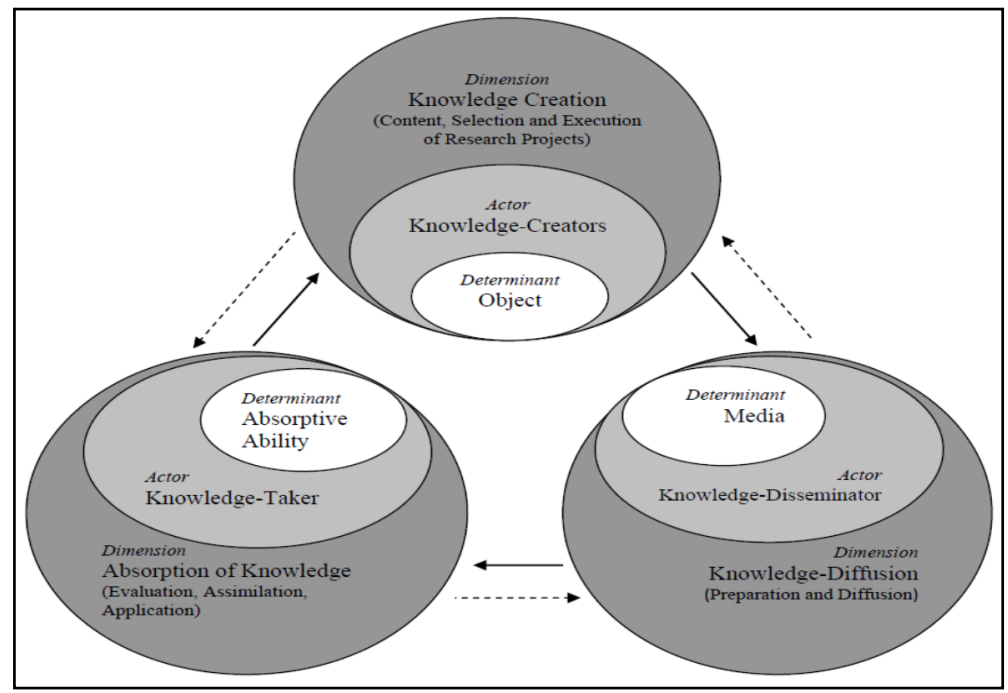

Figure 2: "The interactive-recursive model" of knowledge exchange [4]. 


\section{Mapping the geography of innovation}

Based on Battaglia and Gabrielle [7], cities have become pivotal driving forces of development, locally in terms of regeneration of depressed areas and marginalized sectors and globally for regional competitiveness and trans-border cooperation. Cities are techno-hubs of global flows and they play the role of centres of innovation, as well as of new production and creativity regulated by relations of competitiveness and cooperation.

\subsection{The drivers of innovative regions}

Simply drafting a technology-focused economic vision is not enough, however. A successful vision will also explicitly use technology to pursue three key drivers for any healthy economy.

1. Productivity: A city's economic vision must support aggregate economic growth and promote efficiencies throughout the public and private sectors.

2. The second is inclusivity: A vision must support opportunity for all firms and citizens. Broadband is equally important to the future economic success of households as well as its businesses.

3. The third is resiliency: A vision must support a more sustainable built environment.

\subsection{Innovation hubs vs innovation islands}

According to Locus growth model, which is conceived as a theoretical one, the mechanism driving the emergence of "innovation islands" is due to the average human capital in society or area: people enjoy positive spill overs from fellow members of the same social group, thus becoming more productive themselves. In an environment that is endowed with fluent interpersonal relationships, where people trust each other, tolerance for diversity enhances creativity, and governance of cultural and natural institutions is able to properly manage public endowments, people are expected to gain more than proportionally in productivity. Other economic academics proposed the concept of "innovation hubs ", which may be compared to islands of innovation, but the main difference between these two concepts lies in the consistently higher degree of connectivity of the former vis-àvis the latter.

To provide a socio-economically sustainable development for these regions, Hardman and Lange [8] advocate the creation of innovation centres and science parks, playing the role of convivial epicentres without walls. Such locations will welcome the physical community in a creative environment and connect it, virtually, to the rest of the world.

\subsection{Global innovation "heat map"}

Compiled by management consultancy McKinsey with the World Economic Forum, researchers used 700 variables including infrastructure, demand, government regulation, human capital and business environment to assess the 
activity and impact of hundreds of innovation hubs around the world. Although it's depending basically on the ratio of patent growth within clusters, still this map is considered one of the most comprehensive trials to illustrate the dynamics of global innovation. The mapping system depended on categorizing the "innovative regions" into one of four different categories: "hot springs", "dynamic oceans", "silent lakes", and "shrinking pools".

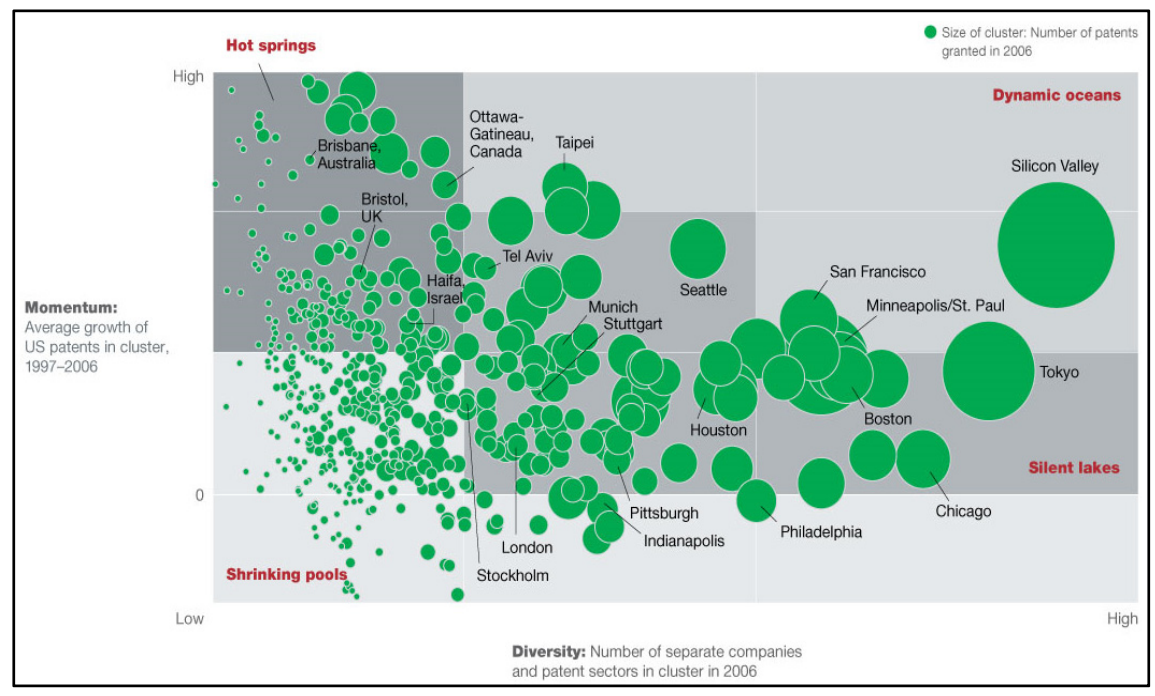

Figure 3: Innovation clusters heat map. Source: Juan Alcacer, Harvard Business School and New York University, McKinsey analysis.

According to Porter [9], the analysis of McKinsey's "innovation heat map" picked out some trends:

1. Political stability and the quality of transport and technical infrastructure have to be in place for any innovation hub to succeed.

2. Hubs tend to develop a specialism and build credibility as an 'innovation hot spring, often around a small number of companies, before expanding.

3. Those hot springs are typically driven by either targeted, government-led investment projects, local benefits such as cheap labour and 'knowledge oases' - places with a rich talent pool like an R\&D department or research university.

4. Further contributing factors are local regulation, local demand or the local investment scene but staffing remains key.

5. As well as the local talent pool, hubs need to be able to attract 'world-class talent'. There are emerging problems in Japan and Europe, for example, because of an aging population, and in the US where highly specialized immigrants are starting to return home. For Asia, the challenge is training their growing populations to world-class level.

6. Survival during a downturn depends on diversification. 


\section{Planning innovation clusters: the eco-system}

At their core, clusters are simply a geographically proximate group of interconnected companies and associated institutions in a particular field, linked by various commonalities and complementarities. What is not simple, however, understands what gives clusters a competitive critical mass.

Clusters foster the collaboration needed to develop new ideas and bring them to market. In this way, successful clusters significantly improve the return on public investments in R\&D and provide global leadership in key technologies. Recognizing this impact, both advanced and emerging economies are making investments and promulgating polices to encourage cluster development.

\subsection{Why innovation clusters?}

Hardman [10] claims that cluster strategies have surfaced again in economic policy discourse because they have the potential to accelerate regional economic growth and assist with the nation's needed economic restructuring. A new generation of industries will drive the economic recovery over the next decade, fuelled by long-term changes in technology, society and geopolitics. The recession was not only a point of change; many argue that it has acted as a catalyst for growth.

This introduces a new paradigm involving three fundamental shifts in the thinking behind innovation clusters as Lange et al. [11]:

-From geography-based to community-driven entities: Instead of viewing science parks as defined geographies, they will be characterized as digital communities of interest where coherence is generated through intellectual proximity rather than just geographic proximity.

-From locally processed innovation to open, borderless innovation: the "wisdom of crowds" elevates relevant new ideas to wider relevant audiences. These community interactions reveal areas of mutual interest that otherwise would not have been identified, so promoting greater uptake of innovative new partnerships to stimulate and accelerate economic growth and wealth creation.

-From technology-driven to technology-enabled spaces: Intelligent and connected buildings, digital collaboration platforms and cloud computing can dramatically change the working space and bring both innovation and productivity gains.

Based on Wessner and Wolff [12], innovation clusters need to draw on the power of an interrelated "quad" of sectors: public, private, civil, and academic to foster a sustainable economic growth.

\subsection{How to integrate innovation clusters in regional development}

There are few economic development policies as popular as clusters. It is hard today to find a country, region, or even city that is not trying to develop a network of complementary and competitive firms. The political appeal is obvious, particularly now that the world's economic crisis has put a spotlight on innovation to diversify economies and create jobs. However, the difficulty lies in turning a 
newly announced "science park" or "hi-tech corridor" into a genuinely competitive centre for innovation.

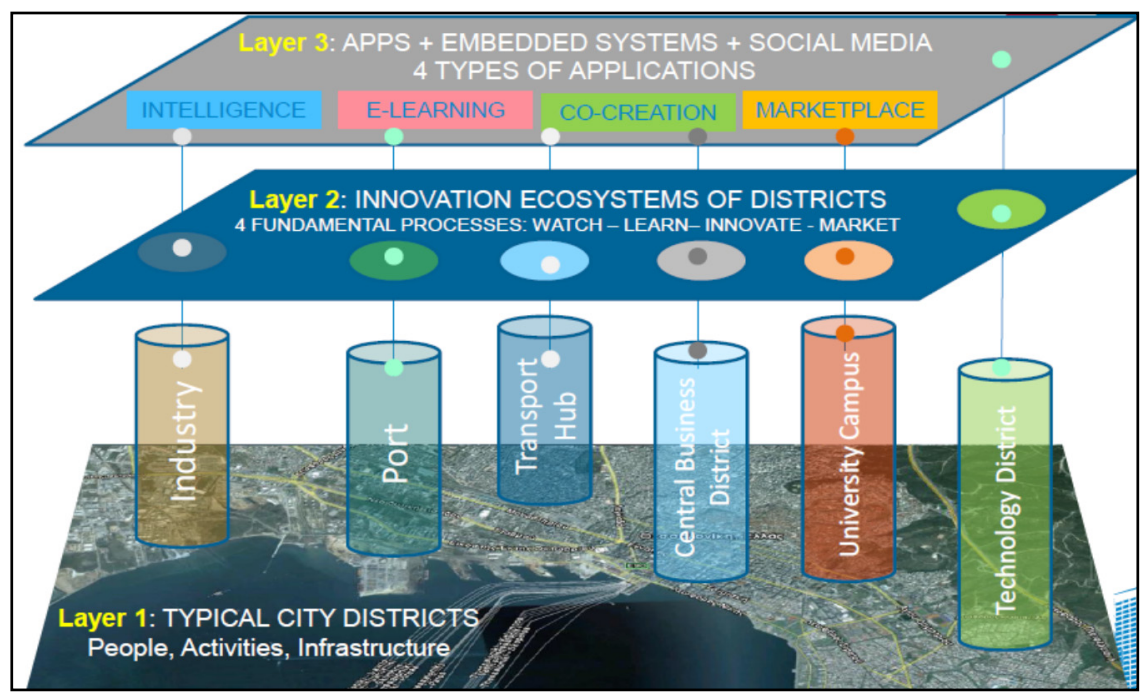

Figure 4: Three Layers of intelligent city. Source: Komninos, Nicos, "The architecture of intelligent cities" URENIO Research Unit.

Successful geographic clusters create, retain and support a self-renewing critical mass, famously exemplified by Silicon Valley and within the UK, the Cambridge IT and Biotech cluster. These dynamic innovation ecologies, "technopoles", evolve as key players change the underlying characteristics of the location and hence the relative competitiveness of a region. The effectiveness of the region's ability to manage and develop its Intellectual Capital (a measure of assets related to knowledge creation) such as: Social Capital; Human Capital; Organizational Capital, Financial Capital and Technology Capital.

\subsection{Integrating innovation clusters within urban level}

According to Nicos [13], the integration of innovative clusters and digital cities determine the building blocks of an intelligent city. An intelligent city is a multiplayer territorial innovation system, integrating knowledge-intensive activities, institutions for cooperation and distributed innovation, and digital communication infrastructure and tools to maximize the innovation and knowledge management capability. It is a 3-level structure:

Layer 1: The basic level of an intelligent city is the city's innovative clusters, in manufacturing and services. This level gathers the creative class of the city made by knowledgeable and talented people, scientists, artists, entrepreneurs, venture capitalists and other creative people, determining how the workplace is organized and how the city is developing. 
Layer 2: A second level is made of institutional mechanisms regulating knowledge flows and co-operation in learning and innovation. This level gathers institutions enhancing innovation: R\&D, venture capital funds, technology transfer and training centres, intellectual property, spin-off incubators, technology and marketing consultants.

Layer 3: Is made up by information technology and communication infrastructures, digital tools and spaces for learning and innovation. These technologies create a virtual innovation environment, based on multimedia tools, expert systems, and interactive technologies, which facilitate market and technology intelligence, technology transfer, spinoff creation, collaborative new product development, and process innovation.

\section{MENA region as an innovation hub: challenges and potentials}

The EU Commission [14] stated that MENA's (Middle East and North Africa's) technology scene is in its infancy and the potential for growth is enormous particularly in the population dense country of Egypt and amongst wealthy consumers in Saudi Arabia. The challenge is not finding the market but rather how to drum up initial funding, where to turn for a supportive ecosystem and an overwhelming need to create legal structures to support early stage start-ups. But like many areas of the world, technology is seen as a risky play with more traditional investments (real estate in particular) showing better returns with less risk.

\subsection{Positioning MENA region within global innovation map}

In the global picture of innovation that emerges from the Global Innovation Index 2013, the Middle East shows continued strengths - in particular through the Gulf Cooperation Council (GCC) countries - as well as intra-regional differences.

As expected, building a global hub for R\&D in the Middle East will not happen overnight, not least because of the need for a strong supply of local talent. But the fragile economies of many advanced countries create an opportunity here.

Top scientists, researchers and entrepreneurs are highly mobile, while immigration policies in many countries are being tightened. With sufficient incentives, this global diaspora of talent could be attracted to help accelerate cluster development.

\subsection{Knowledge-based eco-system and innovation culture, MENA model}

Collaboration between universities, corporate and other members of an innovation ecosystem is often most fluid and effective when the ecosystem members are located in a geographical cluster. MENA countries started very early in constructing science and technology parks and research centres, which can be classified into four groups of clusters: 
- ICT Clusters: techno valley (Riyadh, Dhahran, Doha, Dubai);

- Media Cluster: Egyptian Media Production City, Dubai Media City (UAE);

- Research \& Education Cluster: King Abdullah University of Science \& Technology (KAUST) in Theuwal (Saudi Arabia), Qatar Science \& Technology Park;

- Manufacturing \& Logistics Cluster: hundreds of industrial parks all over the region.

Despite of the highly equipped infrastructure and the ease of technology reach for governmental initiations and private sector, still, missing here is "Collaboration \& Human Resources". One of the flourishing evidences that collaboration is highly possible in this region, an emerging project for electrical connection among MENA countries and South Europe region is considered as a major success story for regional collaboration in this area (Figure 5).

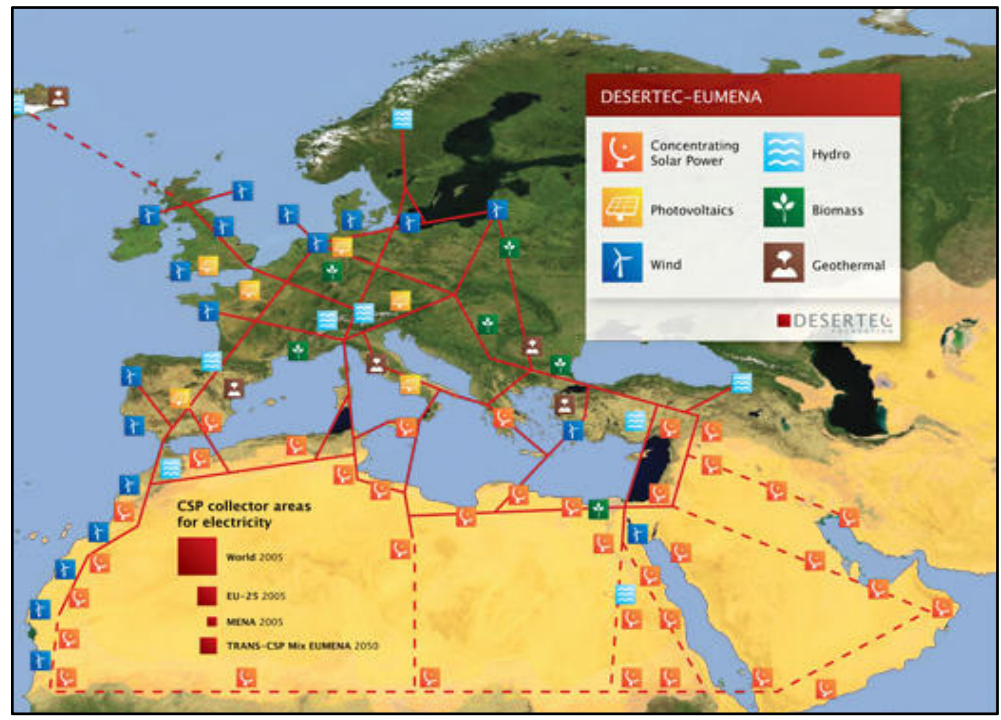

Figure 5: The regional project of electrical connection MENA \& Europe. Source: Deserte.

\subsection{Learned lessons - the building blocks of innovative MENA region}

Based on a comprehensive study for global cases, The Economist [15] stated some ideas might be applicable to the Middle East and North Africa region (MENA) as it seeks to develop its own innovation-led clusters. The key findings are as follows:

- Government has a crucial role to play; so does the market.

- Clusters are about collaboration, not just locating firms in the same place.

- Talent is the single most important factor in developing successful clusters.

- A related focus should be on encouraging the inward migration of talent from around the world. Attracting a star name in a given field can be a crucial catalyst. 
- Governments need to work to promote a culture of innovation and entrepreneurship.

- It is important to create a culture that tolerates different opinions, creativity and risk-taking.

- Clusters work best when they are focused and can compete. Once a specific focus emerges, governments need to identify it, and then work to remove any barriers to competition.

- Governments can do much to create an attractive business environment - and a good place to live.

- A strong local market will help attract R\&D investment, but is not crucial for global success.

- Encourage self-organization, Provide incentives and long-term commitments.

\subsection{Networking innovation clusters in MENA regions - existing islands}

Maintaining a coherent and balanced development for all sub-regions within MENA requires establishing the following pillars to act as a basic foundation for developing the concept of the MENA Knowledge Hubs Master Plan:

- First: Learner centred development, where the learner is a human capital development, the goal of any development tool and knowledge at the same time.

-Second: Knowledge centred development, where a wealth of knowledge that are used as fuel soft renewed vital for the development process.

-Third: HEIs centred development, Higher Education Institutions are the main driver for knowledge production, development and re-generation for sustainable knowledge.

-Fourth: Product centred development, industrial clusters have three basic advantages of clusters which are known as "Marshall's trinity". They are: 1) a pool of skilled labour; 2) knowledge spill overs; and 3) inter-firm linkages.

\section{Concluding remarks}

As a conclusion, the models of innovation clusters and their design principles vary dramatically from case to another. Most developing countries are not replicating the innovation models from developed countries.

Also, the paper highlighted that the major obstacle against fostering innovation in MENA region is collaboration. It highlighted the importance of trust, openness and institutional mechanisms as necessary prerequisites for cooperation to occur between clusters. Institutions are essential to create trust which lie the foundation for open innovation processes.

The development of higher education institute within the city urban context is expected to encourage spreading the culture of innovation and creativity within a sustainable framework specially within developing cities and nations.

For the MENA region, still further studies should explore different perspectives about social equity and sustainable development for the concept of knowledge cities and innovation clusters. 
Although it is not tested yet, this model tends to thrive sustainable development in urban regions by linking between a concentration of human capital and economic growth at the level of city planning, taking into account the level of education as a measure of human capital.

\section{References}

[1] Alexandre Almeida, Cristina Santos, Mário Rui Silva, Bridging science to economy: the role of science parks and technology parks in innovation strategies in "follower" regions, Universidade do Porto, Faculdade de Economia do Porto, FEP Working Papers, pp. 5, 2008.

[2] Enright, Michael J., Roberts, Brian H., "Regional Clustering in Australia", Australian Journal of Management, Vol. 26, Special Issue, 2001.

[3] Gibson, D. V.; Rogers, E. M. "R\&D collaborations on trial. The microelectronics and computer Technology Corporation". Boston MA, Harvard Business School Press, 1994.

[4] Verena Christiane Eckl, "Creating an Interactive-Recursive Model of Knowledge Transfer", DRUID society, 2012.

[5] De Prato, G. and Nepelski, D. 'Mapping the European ICT Poles of Excellence. The Atlas of ICT Activity in Europe', JRC Scientific and Policy Reports EUR 26579 EN. Seville: JRC-IPTS, 2014.

[6] Bozeman, B., "Technological transfer and public policy: a review of research and theory". Research Policy 29(4-5): pp. 627-655, 2000.

[7] Battaglia, Angélo and Gabrielle, Diane. "22@ and the Innovation District in Barcelona and Montreal: a process of clustering development between urban regeneration and economic competitiveness", Research note, 2011.

[8] David J Hardman, Anne Lange, "Science Parks without Walls: Catalysts for Knowledge Economy led Urban Regeneration", the triple helix 9 conference, 2011.

[9] Michael Porter, "Clusters, innovation, and competitiveness: New findings and implications for policy", 2008.

[10] David J Hardman, "Science Parks without Walls: Catalysts for Knowledge Economy led Urban Regeneration", 2011.

[11] Anne Lange, Doug Handler, James Vila, "Next-Generation Clusters: Creating Innovation Hubs to Boost Economic Growth." Cisco Internet Business Solutions Group, 2010.

[12] Wessner CW, Wolff AW, editors. "Rising to the Challenge: U.S. Innovation Policy for the Global Economy" Washington (DC): National Academies Press (US); 2012.

[13] Nicos, Komninos, "What makes cities smart?" URENIO Research, SC Conference, Edinburgh, 2011.

[14] EU Commission, "Next generation innovation policy the future of EU innovation policy to support market growth", 2010.

[15] The Economist, "Fostering innovation-led clusters a review of leading global practices", the Economist Intelligence Unit, 2011. 